\title{
A CRITERION FOR PERRON INTEGRABILITY
}

\author{
D. N. SARKHEL
}

\begin{abstract}
It is shown that a measurable function $f: I=[a, b] \rightarrow R_{e}$ is necessarily Perron integrable if there exists at least one pair of functions $u, l$ : $I \rightarrow R$ such that (i) $u(x-)<u(x)<u(x+)$ and $l(x-)>l(x)>$ $l(x+)$ on $I$, (ii) $I \backslash\left(E_{1} \cup E_{2}\right)$ is countable, where $E_{1}=\left\{x \mid D_{-} u(x)>\right.$ $\left.-\infty, D^{-l} l(x)<\infty\right\}$ and $E_{2}=\left\{x \mid D_{+} u(x)>-\infty, D^{+} l(x)<\infty\right\}$, and (iii) $\max \left\{D_{-} u(x), D_{+} u(x)\right\}>f(x)>\min \left\{D^{-} l(x), D^{+} l(x)\right\}$ a.e. on $I$. In the special case when $u$ and $l$ are respectively major and minor functions of $f$ in the sense of H. Bauer, the result was proved by J. Marcinkiewicz.
\end{abstract}

The purpose of this note is to prove the following theorem.

TheOREM. A measurable function $f: I=[a, b] \rightarrow R_{e}$ is Perron integrable (P-integrable) if [and only if] there exists at least one pair of functions $u, l$ : $I \rightarrow R$ such that (i) $u(x-) \leqslant u(x) \leqslant u(x+)$ and $l(x-) \geqslant l(x) \geqslant l(x+)$ on $I$, (ii) $I \backslash\left(E_{1} \cup E_{2}\right)$ is countable, where $E_{1}=\left\{x \mid D_{-} u(x)>-\infty, D^{-} l(x)\right.$ $<\infty\}$ and $E_{2}=\left\{x \mid D_{+} u(x)>-\infty, D^{+} l(x)<\infty\right\}$, and (iii) $\max \left\{D_{-} u(x)\right.$, $\left.D_{+} u(x)\right\} \geqslant f(x) \geqslant \min \left\{D^{-} l(x), D^{+} l(x)\right\}$ a.e. on $I$.

(Convention: for any $g: I \rightarrow R, g(a-)=g(a)$ and $g(b+)=g(b)$.)

The extent of the Theorem will be clear from the following discussion if we compare the pair of functions $u, l$ with pairs of major and minor functions defined by various authors.

A major function $u$ of $f$ defined by Bauer [1] is continuous and fulfils the condition $-\infty \neq \underline{D} u(x) \geqslant f(x)$ everywhere, that defined by Hake [2] is continuous and fulfils the condition $-\infty \neq D_{+} u(x) \geqslant f(x)$ everywhere, that defined by Saks [4] is not necessarily continuous but fulfils the condition $-\infty \neq \underline{D} u(x) \geqslant f(x)$ everywhere, and that (a right major, to be precise) defined by McShane [3] is continuous and fulfils the condition $-\infty \neq$ $D_{+} u(x) \geqslant f(x)$ nearly everywhere. Unlike others, McShane defines Pintegrability in terms of tetrads of both right and left major and minor functions (defined analogously). Regarding Saks' definition, we first observe

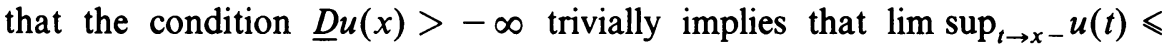
$u(x) \leqslant \lim \inf _{t \rightarrow x+} u(t)$. On the other hand, if $u$ is to be a major function of a P-integrable function, then $u(x-)$ and $u(x+)$ must exist finitely everywhere, since the corresponding indefinite integral $(F$, say) is continuous

Received by the editors June 7, 1977.

AMS (MOS) subject classifications (1970). Primary 26A39.

Key words and phrases. P-integrable, major function, minor function, left major, left minor, right major, right minor, $V B_{*}$. 
and since $u-F$ is nondecreasing. Therefore, we can suppose without loss of generality that $u(x-) \leqslant u(x) \leqslant u(x+)$ everywhere. Similar remarks hold for minor functions.

The Theorem is an improvement of a known result (see [4, (3.13), p. 253]) due to J. Marcinkiewicz which states that a measurable function $f: I \rightarrow R_{e}$ is necessarily $\mathrm{P}$-integrable if it possesses at least one pair of continuous major and minor functions in the sense of Saks; also, the former virtually nullifies Saks' remark [4, p. 253, infra] that in the latter the hypothesis of continuity of the major and minor functions is essential. In support of his remark, Saks considers the functions $f, U, V$ on $[0,1]$ defined by $f(x)=-1 / x^{2}$ for $x>0$ and $f(0)=0, V(x)=1 / x$ for $x>0$ and $V(0)=0$, and $U(x) \equiv 0$. He says that $U$ is a major function and $V$ is a minor function of $f$, and yet $f$ is not P-integrable. But, we observe that in no sense can $V$ be called a minor function, since $D^{+} V(0)=\infty=V(0+)$. Indeed, our Theorem implies that this function $f$ cannot have a minor function of any kind.

We mention two implications of the Theorem. Let

$$
\begin{gathered}
U_{-}(f, I)=\{u: I \rightarrow R \mid u(a)=0, u(x-) \leqslant u(x) \leqslant u(x+) \text { on } I, \\
\left.D_{-} u(x)>-\infty \text { n.e. on } I, D_{-} u(x) \geqslant f(x) \text { a.e. on } I\right\}, \\
L^{-}(f, I)=\left\{l: I \rightarrow R \mid-l \in U_{-}(-f, I)\right\}, \\
U_{+}(f, I)=\{u: I \rightarrow R \mid u(a)=0, u(x-) \leqslant u(x) \leqslant u(x+) \text { on } I, \\
\left.D_{+} u(x)>-\infty \text { n.e. on } I, D_{+} u(x) \geqslant f(x) \text { a.e. on } I\right\}, \\
L^{+}(f, I)=\left\{l: I \rightarrow R \mid-l \in U_{+}(-f, I)\right\} .
\end{gathered}
$$

Call a member of $U_{-}(f, I)$ a left major (of $f$ ), that of $L^{-}(f, I)$ a left minor, that of $U_{+}(f, I)$ a right major, and that of $L^{+}(f, I)$ a right minor. Then the theory of the P-integral can be developed equivalently and directly in terms of these left major and left minor functions and, also, in terms of these right major and right minor functions. (In either case, $u-l$ is nondecreasing, the continuity of the indefinite integral $F$ can be proved easily by using the inequalities $u(x-) \leqslant u(x) \leqslant u(x+), l(x-) \geqslant l(x) \geqslant l(x+)$ and $l(x)$ $\leqslant F(x) \leqslant u(x)$, and the differential property of $F$ can be proved by using first obvious modifications of the usual method and then appealing to the Denjoy relations between the derivates.) Both these approaches unify and simplify the approaches of the authors cited above. In particular, following either of these two approaches and using the Theorem, McShane's direct proof of the theorem on P-integration by parts $[3,65.1$, p. 332] can be shortened considerably.

Proof of The Theorem. Plainly (i) implies that $u(x-)<\infty, u(x+)>$ $-\infty, l(x-)>-\infty$ and $l(x+)<\infty$. Also, using (i), (ii) and (iii), and using minor modifications of well known methods (consult, e.g., [3, 34.1, 57.4] and $[4$, p. 203, supra]), it is readily proved that $u-l$ is nondecreasing. Noting these and referring to the hard core of the proof of Theorem (3.13) [4, p. 253] and to Theorem (7.1) [4, p. 229], it is enough to show that the interval $I$ can 
be expressed as the union of a countable family of sets on each of which $u$ is $V B_{*}[4$, p. 228].

To this end, we first observe that, for every $x \in E_{1}$ there is a positive integer $n$ such that

$$
u(x)-u(y)>-n(x-y) \text { for all } y \in(x-1 / n, x)
$$

and

$$
l(x)-l(y)<n(x-y) \text { for all } y \in(x-1 / n, x) .
$$

Since the function $w=u-l$ is nondecreasing, it follows from (1) and (2) that, for all $y \in(x-1 / n, x)$,

$$
\begin{aligned}
-n(x-y)- & w(x)+w(y) \\
& <u(x)-u(y)=l(x)-l(y)+w(x)-w(y) \\
& <n(x-y)+w(x)-w(y),
\end{aligned}
$$

that is,

$$
|u(x)-u(y)|<n(x-y)+w(x)-w(y) .
$$

Similarly, for every $x \in E_{2}$ there is a positive integer $n$ such that, for all $y \in(x, x+1 / n)$,

$$
|u(y)-u(x)|<n(y-x)+w(y)-w(x) .
$$

Now, let

$$
v(x, y)=|u(x)-u(y)|+w(x)-w(y),
$$

and, for each positive integer $n$, let

$$
A_{n}=\left\{x \in I^{0} \mid v(y, x)<n(x-y) \forall y \in(x-1 / n, x)\right\}
$$

and

$$
B_{n}=\left\{x \in I^{0} \mid v(x, y)<n(y-x) \forall y \in(x, x+1 / n)\right\} .
$$

Then, by hypothesis (ii) and by the preceding observations, it is clear that the set $I \backslash \cup_{n=1}^{\infty}\left(A_{n} \cup B_{n}\right)$ is countable. Therefore, the desired result will be proved if we can show that, for every $n$ and for every closed interval $J$ of length less than $1 / n, u$ is $V B_{*}$ on each of the sets $A_{n} \cap J$ and $B_{n} \cap J$.

Because of symmetry, we give the proof for $A_{n}$ only.

If $y^{\prime}, y \in\left[x^{\prime}, x\right] \subset J$ where $x^{\prime}, x \in A_{n}$, then we have

$$
\begin{aligned}
\mid u\left(y^{\prime}\right)- & u(y)|\leqslant| u\left(y^{\prime}\right)-u(x)|+| u(x)-u(y) \mid \\
& <n\left(x-y^{\prime}\right)+w(x)-w\left(y^{\prime}\right)+n(x-y)+w(x)-w(y) \\
& \leqslant 2\left\{n\left(x-x^{\prime}\right)+w(x)-w\left(x^{\prime}\right)\right\},
\end{aligned}
$$

whence it follows at once that $u$ is $V B_{*}$ on $A_{n} \cap J$.

\section{REFERENCES}

1. H. Bauer, Der Perronsche Integralbegriff und seine Beziehung zum Lebesgueschen, Monatsh. Math. Phys. 26 (1915), 153-198.

2. H. Hake, Uber de la Vallée Poussins Ober- und Unterfunktionen einfacher Integrale und die 
Integraldefinition von Perron, Math. Ann. 83 (1921), 119-142.

3. E. J. McShane, Integration, Princeton Math. Series, 7, Princeton Univ. Press, Princeton, N. J., 1944.

4. S. Saks, Theory of the integral, Monografie Mat. Series, vol. VII, 2nd rev. ed. PWN, Warsaw, 1937; reprint, Dover, New York, 1964. MR 29 \# 4850.

Department of Mathematics, Universtty of Kalyani, Kalyan,, West Bengal, India 